\title{
NEW INEQUALITIES FOR PRODUCTS OF CROSS-SECTION MEASURES
}

\author{
Horst MARTINi AND ZOKHRAB MUSTAFAEV
}

Abstract. The main purpose of this paper is to sharpen some upper and lower bounds on products of cross-section measures of centered convex bodies. The bounds are given in terms of relative inner and outer radii of isoperimetrices of normed spaces, and improve previously known results in the symmetric case. Thus, our results mainly refer to the geometry of finite dimensional real Banach spaces.

Mathematics subject classification (2010): 46B20, 52A20, 52A21, 52A40.

Keywords and phrases: Affine isoperimetric inequalities, cross-section measures, inner radius, intersection body, isoperimetrix, Minkowski geometry, outer radius, projection body.

\section{REFERENCES}

[1] N. S. Brannen, Volumes of projection bodies, Mathematika 43 (1996), 255-264.

[2] H. Busemann, A theorem on convex bodies of the Brunn-Minkowski type, Proc. Nat. Acad. Sci. USA 35 (1949), 27-31.

[3] R. J. Gardner, Geometric Tomography, second edition, Encyclopedia of Mathematics and its Applications 58, Cambridge University Press, New York, 2006.

[4] P. Goodey And W. Weil, Zonoids and generalizations, In: Handbook of Convex Geometry, vol. B, pp. 1297-1326, North-Holland, Amsterdam et al., 1993.

[5] E. LutwaK, Intersection bodies and dual mixed volumes, Adv. Math. 71 (1988), 232-261.

[6] E. Lutwak, On a conjectured projection inequality of Petty, Contemp. Math. 113 (1990), 171-182.

[7] E. LutwaK, Selected affine isoperimetric inequalities, In: Handbook of Convex Geometry, vol. A, pp. 151-176, North-Holland, Amsterdam et al., 1993.

[8] H. MARTINI, On inner quermasses of convex bodies, Arch. Math. 52 (1989), 402-406.

[9] H. MARTINI AND Z. MustafaEV, Some applications of cross-section measures in Minkowski spaces, Period. Math. Hungar. 53 (2006), 185-197.

[10] H. Martini And Z. Mustafaev, On unit balls and isoperimetrices in normed spaces, Colloq. Math. 127 (2012), 133-142.

[11] C. A. Rogers And G. C. Shephard, Convex bodies associated with a given convex body, J. London Math. Soc. 33 (1958), 270-281.

[12] C. SARoglou, Volumes of projection bodies of some classes of convex bodies, Mathematika 57 (2011), 329-353.

[13] R. SCHNEIDER, Stability for some extremal properties of the simplex, J. Geom. 96 (2009), 135-148.

[14] R. SCHNEIDER, Convex Bodies: The Brunn-Minkowski Theory, second edition, Encyclopedia of Mathematics and its Applications 151, Cambridge University Press, 2014.

[15] R. Schneider And W. Weil, Zonoids and related topics, In: Convexity and its Applications, pp. 269-317, Birkhäuser, Basel, 1983.

[16] J. E. SPingarn, An inequality for sections and projections of convex sets, Proc. Amer. Math. Soc. 118 (1993), 1219-1224.

[17] A. C. Thompson, Minkowski Geometry, Encyclopedia of Mathematics and its Applications 63, Cambridge University Press, 1996.

[18] G. Tóth, Notes on Schneider's stability estimates for convex sets in Minkowski spaces, J. Geom. 104 (2013), 585-598. 